\title{
A Queuing Analysis of Packet Dropping over a Wireless Link with Retransmissions
}

\author{
Rachid El Azouzi and Eitan Altman \\ INRIA B.P 93, 2004 Route des Lucioles \\ 06902 Sophia Antipolis Cedex France \\ \{relazouz, altman\}@sophia.inria.fr
}

\begin{abstract}
One of the most important performance measure for real time applications is the packet loss probability. In a wireless environment losses are first due to noisy channels (fading, shadowing etc). In order to improve the radio link, one often retransmits packets that have not been well received (using the Automatic Retransmission reQuest - ARQ). This however may lead to queuing phenomena due to retransmissions and to losses of packets due to buffer overflow. We present a queuing analysis in order to compute the combined effect of the noisy channel, the retransmissions and the buffer overflow. We use advanced spectral methods of quasi-birth and death process in order to analyze the system for general multistate Markov channels and provide an approximation approach based on the theory of singular perturbation.
\end{abstract}

\section{Introduction}

The study of the performance of wireless channels and their effect on the quality of service is a major research issue as it provides tools for design of link layer protocols and dimension link layer buffers, see e.g. [12,13] and references therein. We study in this paper wireless channels that use retransmission of lost packets: the Automatic Retransmission reQuest (ARQ) protocol. We note however that retransmissions cause extra delay which results in larger queues and therefore in possible losses due to buffer overflow. In order to study both the delay statistics as well as the global loss process, we provide a queuing analysis of the radio link.

Our starting point is a link model provided in [12]. The radio channel considered in [12] is modeled using a two state Markov chain: a perfectly "good" state in which transmission succeeds with probability one, and a perfectly "bad" state in which transmission fails with probability one. Our first goal is to allow for a much more rich class of models for the channel state. The need for richer models is motivated by the following considerations:

- It has been shown in $[9,11]$ that more than two states are needed for describing some radio channels; in fact it was shown that one needs typically at least four states.

- Even in the "best" channel state it might be too optimistic too expect losses to occur with probability zero. Conversely, it may be too pesimistic to assume that in a bad state all packets are systematically lost. 
- Assume that for a given packet loss probability $p$ we assign a single state of the channel. This means (if a discrete time model as in [12] is used) that the time during which a packet suffers a loss probability of $p$ has a geometric distribution. If we wish to be able to model other distributions then more than one state should be assigned to a given loss probability!

Our analysis approach is based on modeling of the system as a two dimensional Markov chain, where the first corresponds to the channel state and the second to the number of packets queued at the buffer. In order to obtain the steady state distribution of this system we make use of recent advanced spectral methods of analysis of quasi birth and death (QBD) processes [5,6]. These methods allow us to represent the steady state solution as a simple function of the eigenvectors and eigenvalues of some matrices. For the case that the channel is described by a two dimensional chain, we obtain an explicit solution which has not been available in [12] even for the simpler special case of "perfectly" good and "perfectly" bad channel states.

The second objective of the paper is to study a singular perturbation solution approach for approximating the steady state solution. This approach allows to obtain simple approximations for the case that the channel state evolves much slower than the queue size. In fact, if the speed ratio is given by a parameter $\epsilon$, then the singular perturbation approach allows to represent the steady state probabilities as a Taylor series of $\epsilon$. The first term in the series already gives a very good approximation when $\epsilon$ is sufficiently small, and this term is much simpler to compute than by using the previous exact approach.

The structure of the paper is as follows. We present the model in section 2 . Section 3 provides a detailed spectral analysis and give the steady state channelqueue length for the finite queue model. We concluded by sample numerical results. In section 4 We study a singular perturbation solution approach for approximating the steady state solution and we compare the two methods presented in section 3 and section 4 by numerical results.

\section{Model}

We consider the following queuing system. In each slot, a packet is generated at the transmitter according to a Bernoulli process with arrival probability $p>0$. The channel state can be described by the variable $l \in E:=\{1,2, . ., L\}$, where $l=i$ denote that the packet can be transmitted with probability $q_{i}$, with $q_{i} \in$ $[0,1]$. If a transmission fails we assume that it will be attempted again in the next time unit. As in the first model studied in [12], we assume that the number of retransmissions of a packet by the ARQ protocol is not bounded: retransmissions will be attempted until a success.

$$
\Lambda=\left(\begin{array}{cccc}
\lambda_{11} & \lambda_{12} & \cdots & \lambda_{1 L} \\
\lambda_{21} & \lambda_{21} & \cdots & \lambda_{2 L} \\
\vdots & \vdots & \vdots & \vdots \\
\lambda_{L 1} & \lambda_{22} & \cdots & \lambda_{L L}
\end{array}\right)
$$


be the transition matrix for the error process, where $\sum_{j=1}^{L} \lambda_{i j}=1$. We shall denote $\nu=\left[\nu_{1}, \nu_{2} . ., \nu_{L}\right]$ the probability distribution of the transition matrix $\Lambda$. We assume throughout that $\Lambda$ is irreducible and aperiodic.

The state of the system at time $n$ is described by $X(n)=(l(n), i(n))$, where $l(n)$ is the state of channel in slot $n$ and $i(n)$ is the number of packets in the queue at the beginning of slot $t$. The arrival process in slot $m$ is independent of $X(n)$ for all $m \geq n$. Arrivals are assumed to occur at the end of the slot so that, in particular, they cannot depart in the same slot.

The system has a finite queue of size $B$. A loss occurs whenever an arriving packet finds the queue full. It can be shown that the Markov channel evolution and the assumption of independent arrivals imply that $X(n)$ is a Markov chain with value in a finite state space $\mathcal{B}=\{(i, j), i=1, . . L, j=0, . ., B\}$

Since we consider discrete time, we have to make some convention on the order of events within each time unit. We assume that at each time unit, if the queue is nonempty then first a transmission is attempted, and only if it succeeds then there is a departure; then if there have been an arrival during the slot then it joins the queue. We call this a service-arrival order. This order means in particular that if the queue is full at the beginning of the slot then an arriving packet during the slot need not be lost: it is only lost if the transmission has failed. ${ }^{1}$

The transition probability $L(B+1) \times L(B+1)$ matrix of the Markov chain $X(n)$ are given by

$$
P=\left(\begin{array}{cccccc}
B_{0} & B_{1} & 0 & \ldots & & \\
A_{2} & A_{1} & A_{0} & 0 & \ldots & \\
0 & A_{2} & A_{1} & A_{0} & 0 & \ldots \\
\vdots & \ddots & \ddots & \ddots & \ddots & \\
\vdots & \ddots & \ddots & \ddots & A_{1} & A_{0} \\
0 & \ldots & & 0 & A_{2} & F_{1}
\end{array}\right) \begin{gathered}
0 \\
\vdots \\
B
\end{gathered}
$$

$P$ consists of $B+1$ block rows and block columns. The matrices $B_{0}, B_{1}, A_{0}$, $A_{1}, A_{2}$, and $F_{1}$ are all $L \times L$ non-negative matrices, where $B_{0}=\bar{p} \Lambda, B_{1}=$ $p \Lambda, A_{0}=\operatorname{diag}\left(p \bar{q}_{i}, i=1,, . ., L\right) \Lambda, A_{1}=\operatorname{diag}\left(p q_{i}+\bar{p} \bar{q}_{i}, i=1, . ., L\right) \Lambda, A_{2}=$ $\operatorname{diag}\left(\bar{p} q_{i}, i=1, . ., L\right) \Lambda$ and $F_{1}=\operatorname{diag}\left(p q_{i}+\bar{q}_{i}, i=1, . ., L\right) \Lambda$. Since $\Lambda$ is irreducible and aperiodic, and since $p>0$, the Markov chain $X(n)$ is aperiodic and it contains a single ergodic class. Hence it has a unique stationary (or steady state) probability distribution defined as

$$
\pi(i, j)=\lim _{n \rightarrow \infty} \operatorname{Pr}(l(n)=i, i(n)=j) ; i=1,2, . ., L ; j=0,1, \ldots, B
$$

\footnotetext{
${ }^{1}$ If in contrast, a real system operates in the arrival-service order i.e. first an arrival occurs (and is lost if the queue is full) and then a transmission is attempted, then the expressions for the steady state probability obtained for the service-arrival order are still valid but they apply to the state of the queue just after the arrival occurred in the arrival-service order system. From this it is then easy to derive the steady state distribution at the beginning of a time slot.
} 
The objective of this analysis is to determine the blocking probabilities, the expected delays, and in fact the whole probability distribution $\pi(i, j)$ in terms of the parameters of the system. $\pi(i, j)$ is the unique solution to the equation

$$
\pi=\pi P, \quad \pi \bar{e}_{L}=1
$$

where the notation $\bar{e}_{k}, k \geq 0$ is used to denote a column vector of ones of length $k(B+1)$. For convenience, we partition the stationary vector $\pi$ as $\left[\pi_{0}, \pi_{1}, \ldots, \pi_{B}\right]$, where $\pi_{k}$ of size $1 \times L$, will called the solution vector of level $k, 0 \leq k \leq B$.

\section{Analysis of the System}

\subsection{Spectral Analysis}

In the analysis a special role is played by the number of states of the server for which the transmission probability is zero or one. We denote these numbers by

$$
m_{0}:=\left|\left\{i: q_{i}=1\right\}\right|, \quad m_{2}:=\left|\left\{i: q_{i}=0\right\}\right|
$$

The structure of the transition probabilities would be similar to the one studied [6] if we did not have the first row and column. We handle this problem by reducing the state space, by removing the level zero and in the second phase, we construct the stationary probability of the reduced chain by using the spectral analysis. This will allow us to obtain the steady state probabilities of the original system.

Let $\mathcal{B}_{1}$ be subset of $\mathcal{B}$, where $\mathcal{B}_{1}=\{(i, j) / i=1, . ., L ; j=1, \ldots, B\}$. We define the restricted process $\left\{X_{n}^{0}, n \geq 0\right\}$ as follows: $X_{n}^{0}=X\left(s_{n}\right)$ for $n \geq 0$, so that $X_{n}^{0}$ is the actual state visited when the Markov chain is not for the nth time in level 0 .

Lemma 1. The restricted precess $X_{n}^{0}$ is an irreducible positive Markov chain on the state $\mathcal{B}_{1}$. Its transition is given by

$$
P^{0}=\left(\begin{array}{cccccc}
C 1 & A_{0} & 0 & \ldots & & \\
A_{2} & A_{1} & A_{0} & 0 & \ldots & \\
0 & A_{2} & A_{1} & A_{0} & 0 & \ldots \\
\vdots & \ddots & \ddots & \ddots & \ddots & \\
\vdots & \ddots & \ddots & \ddots & A_{1} & A_{0} \\
0 & \ldots & & 0 & A_{2} & F_{1}
\end{array}\right)\left(\begin{array}{c}
1 \\
\vdots \\
\vdots
\end{array}\right.
$$

where $C_{1}:=A_{1}+A_{2}\left(I-B_{0}\right)^{-1} B_{1}$. Its stationary probability vector is proportional to $\pi^{0}:=\left[\pi_{1}, \pi_{2}, . ., \pi_{B}\right]$, i.e., we have that $\pi^{0} P^{0}=\pi^{0}$.

Proof See Lemma 10.1.1 in [8]. 
The matrix $C_{1}$ records the probability, starting from the level 1 of returning to the level 1 , before reaching the level 2. The lemma implies that the stationary probability vector may be determined by the following equations:

$$
\begin{gathered}
\pi_{0}=\pi_{1}^{0} A_{2}\left(I-B_{0}\right)^{-1}, \pi_{1}^{0}=\pi_{1}^{0} C_{1}+\pi_{2}^{0} A_{2}, \\
\pi_{i}^{0}=\pi_{i-1}^{0} A_{0}+\pi_{i}^{0} A_{1}+\pi_{i+1}^{0} A_{2}, \pi_{B}^{0}=\pi_{B-1}^{0} A_{0}+\pi_{B}^{0} F_{1}, \pi \bar{e}_{L}=1
\end{gathered}
$$

If $\sum_{i=0}^{L} \nu_{i} q_{i} \neq p$, then the steady-state probability vector can be written as a sum of two matrix-geometric terms,

$$
\pi_{j}^{0}=x_{1} R^{j-1}+x_{L} S^{B-j}, \quad j=1,2, . ., B
$$

where the rate matrices $R$ and $S$ are respectively the minimal nonnegative solutions to the quadratic matrix equations (for details see Naomov [5]).

$$
A_{0}+R\left(A_{1}-I\right)+R^{2} A_{2}=0, \quad S^{2} A_{0}+S\left(A_{1}-I\right)+A_{2}=0
$$

Next we provide a simpler representation of (5). To that aim we introduce some definitions and two Lemmas. Define the quadratic matrix polynomials $T(z)$ and $\bar{T}(z)$ :

$$
T(z):=A_{0}+z\left(A_{1}-I\right)+z^{2} A_{2}, \quad \bar{T}(z):=A_{2}+z\left(A_{1}-I\right)+z^{2} A_{0}
$$

The solution (3)-(4) is closely related to the roots and the left nullvector of $T(z)$. Let $(\alpha, u)$ be a root-nullvector pair of $T(\alpha)$, thus satisfying the equation

$$
u T(\alpha)=0, \operatorname{det}[T(\alpha)]=0
$$

We observe that the non-zero roots of $\operatorname{det}[T(z)]$ are the reciprocals of non zero roots of $\operatorname{det}[\bar{T}(z)]$ with the same corresponding left nullvector. We shall analysis the nature of the roots and the nullvectors of $T(z)$

Lemma 2. 1. If $\alpha$ is an eigenvalue of $R$ with algebraic multiplicity $m$, then $\alpha$ is a root of the polynomial $\operatorname{det}[T(z)]$ and its multiplicity is at least $m$. And if $u$ is an eigenvector of $R$ corresponding to the eigenvalue $\alpha$, then $u$ is a left nullvector of $T(\alpha)$.

2. If $\alpha \neq 0$ and $1 / \alpha$ is an eigenvalue of $S$ with algebraic multiplicity $m$, then $\alpha$ is a root of the polynomial $\operatorname{det}[T(z)]$ and its multiplicity is at least $m$. And if $u$ is an eigenvector of $S$ corresponding to the eigenvalue $1 / \alpha$, then $u$ is a left nullvector of $T(\alpha)$.

3. The left nullspace of $R$ and $A_{0}$ coincides, and so do the left nullspace of $S$ and $A_{2}$

Proof See the full of the paper [4].

Remark 1. Recall $m_{0}$ and $m_{2}$ are the number of state $i \in\{1,2, . ., L\}$ for which $q_{i}=1$ and $q_{i}=0$. Then after some permutation of the rows in matrix $A_{i}$, 
$i=0,2$, the matrix $A_{i} i=0,2$ can be writing as the following block triangular matrix $^{2}$

$$
A_{i}=\left(\begin{array}{cc}
A_{i}^{1} & A_{i}^{2} \\
\mathbf{0} & \mathbf{0}
\end{array}\right)
$$

The eigenvalues of $A_{i}, i=0,2$ are those of $A_{i}^{1}$ with those of matrix null $\mathbf{0} \in M_{m_{i}}$, counting multiplicities. Then if $A_{i}^{1}, i=0,2$ has eigenvalue zero with multiplicity $n_{i}, 0$ is eigenvalue of $A_{i}$ with algebraic multiplicity $r_{i}$ where $r_{i}=m_{i}+n_{i}, i=0,2$.

In the sequel, we assume that the sum of all principal minus of $T(z)$ of order $L-n_{0}$ is nonzero. We deduce from these results the following

Lemma 3. (i)- The polynomial $\operatorname{det}[T(z)]$ has a root with geometric multiplicity $r_{0}$ located at $z=0$, and (ii)- $2 L-r_{0}-r_{2}$ roots. For their location we distinguish two cases:

1. If $p<\sum_{i=1}^{L} \nu_{i} q_{i}, L-r_{0}$ of them lie in disc $|z|<1$, one at $z=1$, and $L-1-r_{2}$ in $\operatorname{disc}|z| \geq 1$ with $z \neq 1$.

2. If $p>\sum_{i=1}^{L} \nu_{i} q_{i}, L-1-r_{0}$ of them lie in disc $|z|<1$, one at $z=1$, and $L-r_{2}$ in disc $|z| \geq 1$ and $z \neq 1$

Proof see the full version of the paper [4]

Now, we return to the steady-state for the restricted process $X_{n}^{0}$. It was already showed that the matrices $R$ and $S$ have a full set of eigenvectors. Consequently the expression (5) can thus rewritten in spectral-expansion form :

$$
\pi_{j}^{0}=\sum_{k=1}^{L} \beta_{k}\left(\phi_{k}\right)^{j-1} w_{k}+\sum_{k=L+1}^{2 L} \beta_{k}\left(1 / \phi_{k}\right)^{B-j} w_{k}, 1 \leq j \leq B
$$

with $\phi_{1}, . ., \phi_{L}$ the eigenvalues of the matrix $R$ and $w_{1}, w_{2}, . ., w_{L}$ the corresponding left eigenvectors, and $1 / \phi_{L+1}, . ., 1 / \phi_{2 L}$ are the eigenvalues of $S$ with corresponding left eigenvectors $w_{L+1}, . ., w_{2 L}$. If $m_{2}+n_{2}>0$, then we set the corresponding $\phi_{k}$ equal to $\infty$ and we write $1 / \phi_{k}=0$. Moreover by convention we set $0^{0}=1$.

Here the vectors $\pi_{j}, j=1,2, . ., L$ are know if $\beta_{k}$ 's are known. We derive an alternative set of equations which uniquely determines the coefficients $\beta_{k}$ by combining the relation (10) with the equations (3), (4) and the normalization condition.

\subsection{Examples and Numerical Results}

In the full version of the paper [4], we illustrate how to apply this method to a channel that can be described by two states.

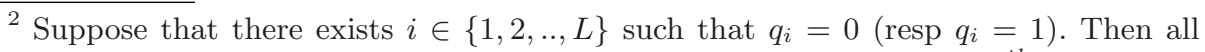
entries in row $i$ of the matrix $S\left(\operatorname{resp} R\right.$ ) equal 0 , so that $1_{i}$ with the $i^{\text {th }}$ entry equal to 1 and all other entries equal to 0 is a left nullvector of $S$ (resp $R$ ), Indeed, when the state of server is $i$ in which $q_{i}=0$, no packet can be transmitted. Hence starting from $(j, i), \forall j$, no state state of level $j-1$ can ever be visited before another state in the level $j$ is visited.
} 


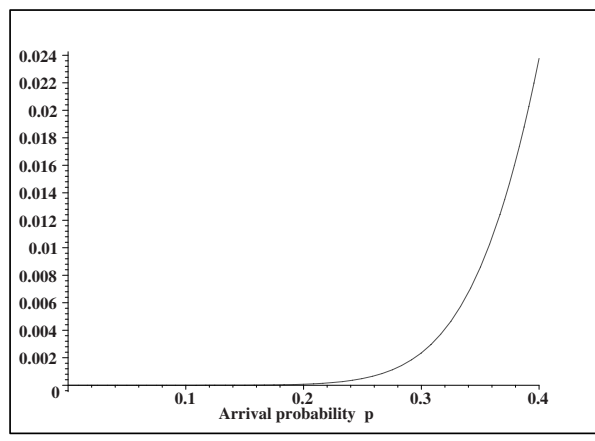

Fig. 1. The dropping probability of a packet as function of the arrauval probability $p$ for $q_{1}=1, q_{2}=0, \lambda_{11}=0.3$, $\lambda_{22}=0.5$ and buffer size $B=6$

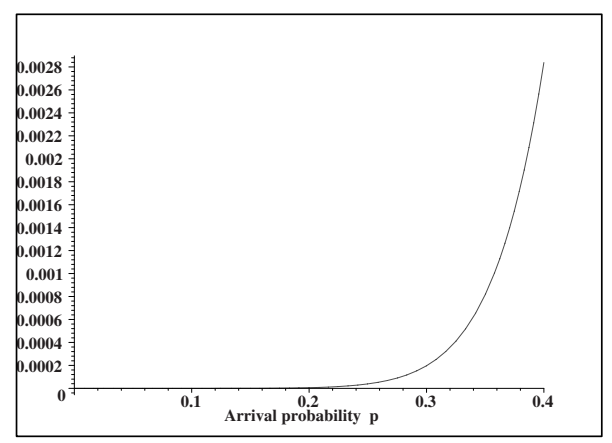

Fig. 2. The dropping probability of a packet as function of the arrival probability $p$ for $q_{1}=1, q_{2}=0.2, \lambda_{11}=0.3, \lambda_{22}=0.5$ and buffer size $B=6$

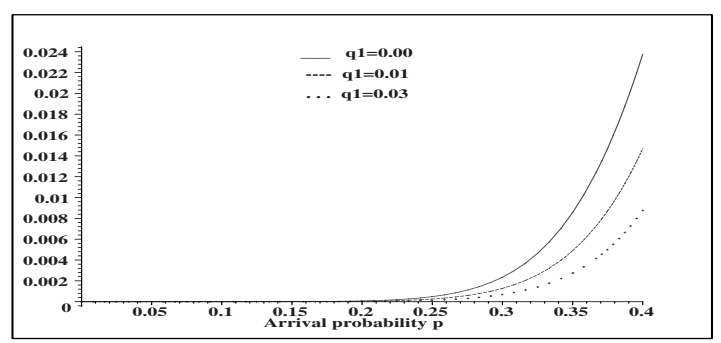

Fig. 3. The dropping probability of a packet as function of the arrival probability $p$ for $q_{1}=1, \lambda_{11}=0.3, \lambda_{22}=0.5$ and buffer size $B=10$ and $q 2=0,0.01,0.03$

Numerical investigation. In this paragraph, we present some numerical results obtained based on the analysis described in previous section 3 . We investigate the relationship between the dropping probability and arrival rate $p$. In particular, we compare the values of the performance measures for two different schemes: The first schemes is perfectly good/absolutly bad state in which the transmission succeeds with probability 1 or 0 , and second scheme is a perfectly good/fairly bad in which the transmission succeeds with probability 1 or 0.2 .

In figures 1 and 2, the dropping probability is plotted versus the arrival rate for first scheme (resp. scheme 2).

As shown in figure 1-2, the performance measures (dropping probability) for two schemes are different. It is true that the modification on the transmission in bad state will influence the performance, but more surprisingly, the difference between the performance measures is of the order $10^{-1}$. This actually confirms that using the simple model of scheme 1 (e.g. $[12,14,13]$ ) does not adequately capture the process of packet errors. In order to verify this, we calculate the performance measures with a small change in the transmission probability $q_{2}$ in the region near 0 for scheme 1 (see figure 3 ), we observe a large effects on 
the performance measures when the arrival rate is more than 0.3. From this observation, it is clear that the values of the transmission probabilities have an important role to model the process packet errors. We conclude that we have to be careful when using the simplify model to represent the wireless the analytic system.

\section{Approximations Using Singular Perturbation}

\subsection{Preliminaries}

Singular perturbation is a powerful approach do obtain simple approximation of steady state probabilities of Markov chains in which we can identify two time scales: there are several group of states such that there are rare transitions between groups and frequent transitions within each group (see e.g. $[2,3,10]$ ) We shall apply this method to the case when the transitions of the queue size occur much more frequently than those of the channel states. This will typically be the case in high speed channels. The singular perturbation approach can also be useful for the opposite case in which the channel states evolve much faster than the queue size process.

We thus represent the transitions of the channel state as $\Lambda(\epsilon)=I+\epsilon \Lambda_{1}$ where $\Lambda_{1}$ is a generator (so that $\Lambda(\epsilon)$ represents indeed transition probabilities) and $\epsilon$ is a small parameter. Hence the transition matrix of the Markov chain $X(n)$ becomes

$$
P(\epsilon)=Q_{0}+\epsilon Q_{1}
$$

where $Q_{0}$ is the probability transition matrix of the unperturbed Markov chain corresponding to strong interaction, and $Q_{1}$ is the generator corresponding to weak interaction, i.e.,

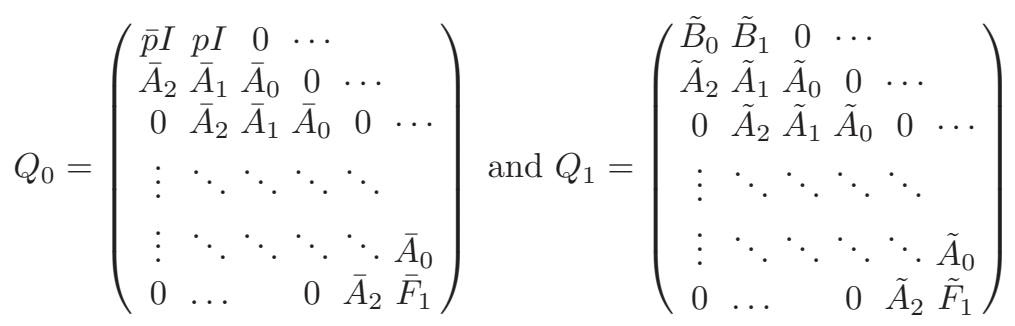

where $\bar{A}_{2}=\operatorname{diag}\left(\bar{p} q_{i}, i=1 . ., L\right), \bar{A}_{1}=\operatorname{diag}\left(p q_{i}+\bar{p} \bar{q}_{i}, i=1 . ., L\right), \bar{A}_{0}=$ $\operatorname{diag}\left(p \bar{q}_{i}, i=1 . ., L\right), \bar{F}_{1}=\operatorname{diag}\left(p q_{i}+\bar{q}_{i}, i=1 . ., L\right), \tilde{B}_{0}=\bar{p}\left(\Lambda_{1}\right), B_{1}=p\left(\Lambda_{1}\right), \tilde{A}_{2}=$ $\operatorname{diag}\left(\bar{p} q_{i}, i=0,1 . ., L\right) \Lambda_{1}, \tilde{A}_{1}=\operatorname{diag}\left(p q_{i} \bar{p} \bar{q}_{i}, i=1 . ., L\right) \Lambda_{1}, \tilde{A}_{0}=\operatorname{pdiag}\left(p \bar{q}_{i}, i=\right.$ $1 . ., L) \Lambda_{1}$, and $\tilde{F}_{1}=\operatorname{diag}\left(p q_{i}+\bar{q}_{i}, i=1 . ., L\right) \Lambda_{1}$.

Let $\pi^{\epsilon}(k, i), i=1 . . L, j=0,1, . ., B$, be the probability distribution of the state of the channel and the number of packets in the system in steady-state. 
This is exactly the perturbation problem under the assumption that there exists a ergodic class (i.e., has exactly one closed communicating set of states), and $Q_{0}$ contains $L$ subchains.

The partition of state set of Markov chain associated with the probability matrix $Q_{0}$ depends of the number of service for which the transmission probability is zero or one. Indeed, if $m_{0}=m_{2}=0$, the $L$ recurrent classes $\xi_{i}, i=1,, L$ are given by

$$
\xi_{i}=\{(i, j), j=0,1, . . B\}
$$

and the unperturbed chain admits no transient state (block diagonal structure). Now if $m_{0}>0$ or $m_{2}>0$, let $E_{B}=\left\{i / q_{i}=0\right\}$ and $E_{G}=\left\{i / q_{i}=1\right\}$. In this case, the $L$ recurrent classes are given by : $\xi_{i}=\{(i, 0),(i, 1)\}$ if $i \in$ $\left\{j / q_{j}=1\right\}, \xi_{i}=\{(i, B)\}$ if $i \in\left\{j / q_{j}=0\right\}$ and $\xi_{i}=\{(i, j) j=0,1, . . B\}$ otherwise. The set of transient states $T$ is given by $T=\left\{(i, j) / j \neq 0,1\right.$ and $q_{i}=$ $1\} \cup\left\{(i, j) / j \neq B\right.$ and $\left.q_{i}=0\right\}$. Without loss of generality, the channel state in which the transmission probability is zero or one, are described by the variable $l \in\left\{1, . ., r_{0}\right\}$ where $r_{0}=m_{0}+m_{2}$.

\subsection{Taylor Series Expansion for Steady State}

The above assumptions still implies that the stationary probability $\pi^{\epsilon}$ has the following property

Lemma 4. The stationary probability $\pi^{\epsilon}$ of the perturbed chain has a Taylor series expansion : $\pi^{\epsilon}=\sum_{n=0}^{\infty} \pi^{(n)} \epsilon^{n}$. Moreover $\pi^{(n)}, n \geq 0$ are recursively calculated as follows:

$$
\begin{aligned}
\pi^{(0)}\left[I-Q_{0}\right]=0, & \sum_{(i, j)} \pi^{(0)}(i, j)=1 \\
\pi^{(n)}\left[I-Q_{0}\right]=\pi^{(n-1)} Q_{1}, & \sum_{(i, j)} \pi^{(n)}(i, j)=0
\end{aligned}
$$

Proof see Schweitzer [10],

We consider the unperturbed Markov chain associated with the transition probability matrix $Q_{0}$. There exists a partition of states set of Markov chain $X(n)$ into of family of $L$ recurrent classes $\xi_{i}, i=1, \ldots, L$. To each class $\bar{\xi}$ is associated the invariant measure (row vector) $m_{\bar{\xi}}$ of the recurrent subchain defined on the class $\bar{\xi} \in \bar{S}=\left\{\xi_{1}, . ., \xi_{L}\right\}$.

Let us consider the natural numbering the states of $\mathcal{B}$ after the grouping defined by the partition $\bar{S}$. With this numbering, the transition probability matrix $Q_{0}$ has the following block structure: 


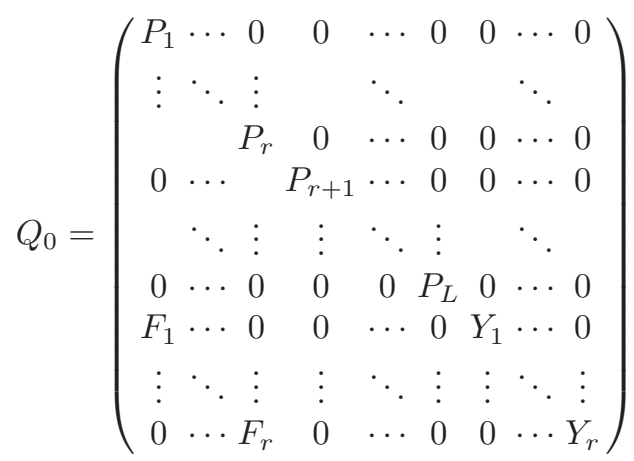

where we distinguish three cases : For $i \notin\{1, . ., r\}, P_{i}$ is a $(B+1) \times(B+1)$ matrix :

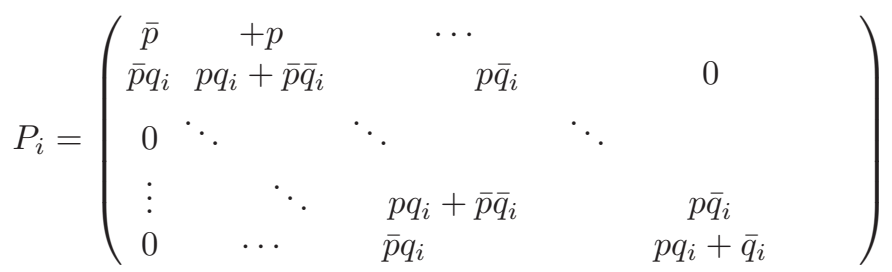

For $i \in E_{G}, P_{i}$ is $2 \times 2$ matrix, $F_{i}$ is $(B-1) \times 2$ and $Y_{i}$ is a $(B-1) \times(B-1)$ matrix :

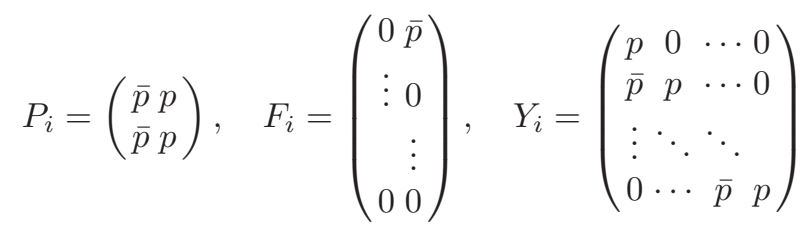

For $i \in E_{B}$, the matrix $P_{i}=1, F_{i}$ is a $B$ vector given by $F^{T}=(0, . ., 0, p)$ and $Y_{i}$ is a $B \times B$ matrix, where

$$
Y_{i}=\left(\begin{array}{cccc}
\bar{p} & p & \cdots & 0 \\
0 & \ddots & \ddots & 0 \\
\vdots & \cdots & \bar{p} & p \\
0 & \cdots & 0 & \bar{p}
\end{array}\right)
$$

Let us define the matrix $\bar{Q}_{1}=\left(\bar{Q}_{1}\right)_{\xi \xi^{\prime}}$ by: $\left(\bar{Q}_{1}\right)_{\xi_{i} \xi_{j}^{\prime}}:=\sum_{k=1}^{B} m_{\xi_{i}}(k)$ $\sum_{k^{\prime}=1}^{B}\left(Q_{1}\right)_{(i, k),\left(j, k^{\prime}\right)}$. We note that $\bar{Q}_{1}$ is a generator of an aggregated Markov chain on a state space $\bar{S}$. Now let $\bar{\nu}=\left[\bar{\nu}_{1}, . ., \bar{\nu}_{L}\right]$ the probability distribution of the aggregated Markov chain. Note in view of definition of $\bar{Q}_{1}$, we observe that the generator of aggregated Markov chain is $\Lambda_{1}$. Hence $\bar{\nu}$ is the unique stationary distribution of ergodic Markov chain $l(n)$, i.e.,

$$
\bar{\nu} \Lambda_{1}=0, \quad \nu \mathbf{1}=1 .
$$


Following Delebecque[3], the unperturbed stationary probability is given by

$$
\pi^{(0)}(i, j)=\bar{\nu}_{i} m_{\xi_{i}}(j)
$$

where $m_{\xi_{i}}$ is the probability distribution of the recurrent class which given by the following lemma.

Lemma 5. For each $i=1,2, . ., L$, the stationary distribution $m_{\xi_{i}}$ of recurrent class $\xi_{i}$ is given by:

1- If $i \notin E_{G} \cup E_{B}, m_{\xi_{i}}(j)=\frac{p^{j}\left(1-q_{i}\right)^{j-1}}{q_{i}^{j}(1-p)^{j}} m_{\xi_{i}}(0), \quad j=1, . ., B$, where $m_{\xi_{i}}(0)=$ $\frac{q_{i}-p}{q_{i}-p \alpha^{B}}$ with $\alpha=\frac{p\left(1-q_{i}\right)}{q_{i}(1-p)}$.

2-If $i \in E_{G}, m_{\xi_{i}}(0)=1-p$ and $m_{\xi_{i}}(1)=p$.

3- If $i \in E_{B}, m_{\xi_{i}}(B)=1$.

Proof see the full version of the paper [4].

We shall denote $q_{\xi_{i}}$ the right eigenvector corresponding to the one eigenvalue of the matrix $P_{i}$. Recall that the right eigenvector provide the probability to end in the class $\xi_{i}$ starting from $i$. In our model, the vector $q_{\xi_{i}}$ is given as follows: For each $j=0,1, . ., B, q_{\xi_{i}}(k, j)=1$, if $k=i$ and $q_{\xi_{i}}(k, j)=0$ otherwise.

Following the analysis in [1, Chap. 3], we can obtain the stationary distribution of the perturbed system by another equivalent system with the matrix coefficients of smaller dimension.

Theorem 1. A solution of the equations (11)-(12) with the normalization conditions is given by the following recursive formulae:

1. $\pi^{(0)}=\bar{\nu} M$, where $\bar{\nu}$ is the stationary distribution of aggregated Markov chain $\Lambda_{1} \in \mathbb{R}^{L+1 \times L+1}$ and $M \in \mathbb{R}^{(L+1) \times(L+1)(B+1)}$ is a matrix whose rows are stationary distribution of the Markov chain $Q_{0}$ and $N \in \mathbb{R}^{(L+1)(B+1) \times(L+1)}$ is a matrix of eigenvectors corresponding to the zero eigenvalue of the unperturbed generator $Q_{0}-I$.

2. $\pi^{(n)}=\pi^{(n-1)} Q_{1} H_{0}+\pi_{1}^{(n)} M, \quad \pi_{1}^{(0)}=\nu, \pi_{1}^{(n)}=\pi_{1}^{(n-1)} M Q_{1} H_{0} Q_{1} N H_{1}$, where $H_{0}$ is a deviation matrix of the unperturbed Markov chain $H_{0}=[I-$ $\left.Q_{0}+N M\right]^{-1}-N M$ and $H_{1}$ is a deviation of the aggregated Markov chain $H_{1}=\left[-\Lambda_{1}+\Lambda^{*}\right]^{-1}-\Lambda^{*}$, where $\Lambda^{*}$ is the ergodic projection of transition matrix $\Lambda-I$, i.e., $\Lambda^{*}=\mu_{L} \nu$, with $\mu_{L}=[1, . ., 1]^{T} \in \mathbb{R}^{L+1}$.

Observe that the stationary distribution is obtained here more efficiently than by using the spectral analysis given in section 3. In this reduced system, we need only to compute the stationary distribution of aggregated Markov chain $\Lambda$ since the stationary distributions of each recurrent class of the unperturbed matrix transition $Q_{0}$ are given by Theorem 1 .

\subsection{Examples and Numerical Results}

Consider the same example as the one presented in subsection 3.2. Also without loss of generality, we assume that $q_{1} \neq q_{2}$. Since the channel state describes by two states, the Markov chain associated with probability matrix $Q_{0}$ has 2 


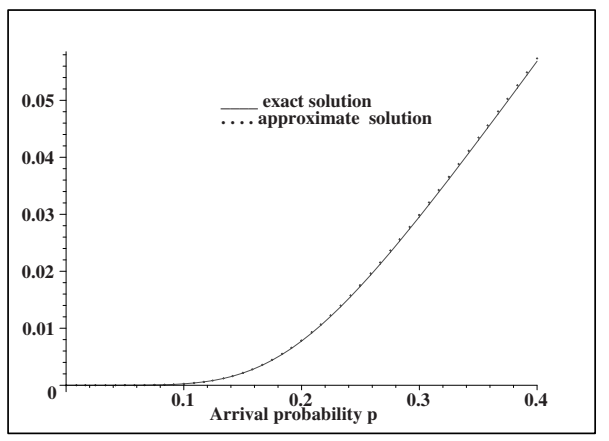

Fig. 4. Dropping probability versus arrival Fig. 5. Dropping probability versus arrival probability for $\epsilon=10^{-3}, a=1 / 5, b=1 / 2$, probability for $\epsilon=10^{-3}, a=1 / 5, b=1 / 2$, $q_{1}=1, q_{2}=0$ and $B=5$. Comparison of $q_{1}=1, q_{2}=0.2$ and $B=30$. Comparison two methods: spectral analysis (exact so- of two methods: spectral analysis (exact solution) and approximate solution.

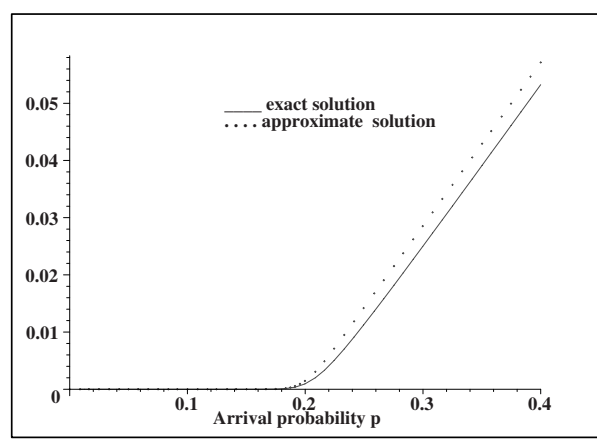

lution) and approximate solution.

recurrent classes and transient states if $m_{0}>0$ or $m_{2}>0$. The stationary probability distribution of the aggregated Markov chain $\Lambda_{1}$ is given by $\bar{\nu}_{1}=$ $\frac{b}{a+b}, \quad \bar{\nu}_{2}=\frac{a}{a+b}$, where $\Lambda_{1}=\left(\begin{array}{c}-a+a \\ b-b\end{array}\right)$

Now, we are going to illustrate how to apply this method and obtain a simple approximation for steady state solution. This approximation can be presented by first term in the Taylor series of $\epsilon$ in which the first term is given by $\pi^{(0)}=$ $\left[\bar{\nu}_{1}, \bar{\nu}_{2}\right]\left[\begin{array}{l}m_{\xi_{1}} \\ m_{\xi_{2}}\end{array}\right]$

In figures 4-5 we compare the dropping probability for various values of the buffer size and for $a=1 / 5, b=1 / 2, q_{1}=1$ and $q_{2}=0.2$, as obtained via the two methods presented in section 3 and section 4 .

From plots in figures 4-5, we observe that the first term in the Taylor series gives a good approximation when $\epsilon$ is small $\left(\epsilon=10^{-3}\right)$. Moreover, we observe that when the buffer size increases the error between the exact solution and the approximation solution increases. An intuitive explanation is as follows: when we use the first term of the Taylor series, we get the limiting behavior as $\epsilon$ tends to zero, i.e. as the transitions of the queue sizes occur much faster than the transitions of the channel state. However, as the buffer size increases, the probability of reaching its boundaries become smaller (it converges to zero when the buffer size converges to infinity for the arrival probability that we chose) which means that the time between transitions to the state of full queue do not occur much faster than the transitions of the channel state. 


\section{References}

1. K. E. Avrachenkov, "Analytic Perturbation Theory and its Applications", Thesis in University of South Australia, 1999. Available at http://www-sop.inria.fr/mistral/personnel/K.Avrachenkov/moi.html

2. P.J. Courtois, "Decomposability: queueing and computer system applications", Academic Press, New York, 1977.

3. F. Delebecque, "A reduction process for perturbed Markov chains," SIAM J. App. Math., vol. 43, pp. 325-350, 1983.

4. R. El Azouzi, and E. Altman, "Queueing Analysis of Link-Layer Losses in Wireless Networks", available at http://www-sop.inria.fr/mistral/personnel/Rachid.Elazouzi/moi.html

5. V. A. Naoumov. Matrix-multiplicative approach to Quasi-Birth-and-Death processes analysis. In: Matrix-analytic Methods in Stochastic Models- Lecture Notes in Pure And Applied Mathematics. Eds. A.S. Alfa and S.R. Chakravarthy. Marcel Dekker, New York (1996), 87-106.

6. R. N. Queija, "Processor-Sharing Models for Intergrated-Service Networks", PhD Thesis, Eindhoven University of Technology, January 2000, ISBN 90-646-4667-8.

7. P. Lancaster, Theory of Matrices, Academic Press, New York, 1969.

8. G. Latouche and R. Ramaswami. "Introduction to Matrix Analytic Method in Stochastic Modeling". Society for Industrial and Applied Mathematics, 1999.

9. M. Rahman, M. Bulmer and M. Wilkinson, "Error models for land mobile satellite channels", Australian Telecommunication Research, Vol. 25 No 2, pp. 61-68, 1991.

10. P.J. Schweitzer, "Perturbation theory and finite Markov chains", J. Appl. Prob., v.5, pp.401-413, 1968.

11. B. Vucetic and J. Du, "Channel modeling and simulation in satellite mobile communication systems", IEEE J. on Selected Areas in Communnications, Vol. 10, No. 8, pp. 1209-1218, 1992.

12. M. Zorzi, "Data-Link dacket dropping models for wireless local communication" IEEE Tran on Vehicular tachnology, vol. 51, No. 4, July 2002.

13. M. Zorzi and R. R. Rao, "Lateness probability of a transmission scheme for error control on a two-state Markov channel", IEEE Tran Commun vol 47, pp. 15371548, Oct, 1999.

14. M. Zorzi, R. R. Rao and L. B. Milstein, Onthe accuracy of a first-order Markov model for data transmission on fading channels: Proc. IEEE ICUPC, pp. 211-215, Nov, 1995. 